\title{
Schema discrimination as a function of training
}

\author{
SELBY H. EVANS AND ED M. EDMONDS
}

TEXAS CHRISTIAN UNIVERSITY

With knowledge of results Ss learned to distinguish between the presence and the absence of a schema and to distinguish between different schemata. Since a schema may be regarded as a statistical concept, the results also show that humans readily learn statistical concepts.

A number of investigators (e.g., Attneave, 1957; Oldfield, 1954; Woodworth, 1938) have suggested that humans organize or encode the redundant (schematic) aspects of stimuli to reduce information processing requirements. That is, given a family of stimuli with common elements, each member is remembered in terms of the common elements (or schema) and the deviations specific to that member.

Attneave (1957) demonstrated that prior familiarization with a prototype faciliated paired-associates learning with deviations from a prototype. In a natural environment, however, there may be no prototype, but only a family of varying instances, from which the schema would have to be abstracted (e.g., there is no "true" shape for a dog). Edmonds \& Evans (1966) have presented evidence that such abstraction can occur when Ss are trained with a set of varying instances representing the same schema. But the natural environment does not present so simple a task; rather, instances representing many schema families are mixed together. If schematic encoding is to be used, Ss must be able to distinguish between members of different schema families and associate each instance with its appropriate schema family; i.e., a schema family corresponds to a statistical concept (Evans, 1964).

The present study was intended to determine whether Ss could readily be trained to distinguish members of a schema family from non-members. The study was also designed to determine whether this distinction was easier with patterns having no schema than with patterns belonging to a different schema family. Since no prototype was provided, Ss again had to abstract the schema from a set of varying instances.

Method

Subjects. The Ss were 40 undergraduates enrolled in psychology courses at Texas Christian University. They were randomly assigned to the two experimental groups which are referred to as $67 \%$ Ss and $0 \%$ Ss.

Patterns. Patterns were produced by a computer program, VARGUS 7 (Edmonds \& Evans, 1966; Evans, 1964; Evans, Edmonds, \& Mueller, 1966) which draws histoform patterns at random from a defined population and allows independent manipulation of schema and redundancy. The average area of the patterns remains unaffected by manipulations of schema and of redundancy. The patterns are generated by mapping a Markov process into a sequence of column heights; the schema is represented by a most probable sequence (MPS) favored by the transitional probabilities. Redundancy is controlled by varying the magnitude of the probability associated with each step of the MPS. In the present experiment, each of four different most probable column height sequences (MPS's), designated patterns set PS1, PS2, PS3, PS4, was used in a Markov process to produce $67 \%$ redundant patterns. A fifth set of $0 \%$ redundant patterns (PS5) was produced by making all column height sequences equiprobable.

Task and procedure. $\quad$ Each $S$ was given a 15 page booklet with three nonidentical patterns printed on each page and he was instructed to select the pattern on each page that was "most different from" the other two patterns. Each page of the booklet given to $67 \% \mathrm{Ss}$ contained two different PS1 patterns and a third pattern chosen at random from PS2, PS3, or PS4. The $0 \%$ Ss received the same PS1 patterns as did $67 \%$ Ss but a different PS5 pattern constituted the third pattern on each page. The position of the third pattern in each three pattern set was randomly determined for both experimental groups. The Ss were allowed $45 \mathrm{sec}$. to choose a pattern on each page; they were then told which pattern was correct.

\section{Results and Discussion}

In Fig. 1 the mean number of correct pattern selections for $0 \%$ Ss and $67 \%$ Ss are plotted as a function of blocks of three trials. Comparisons based on block 5 showed that both experimental groups performed significantly (CR $=4.41, \mathrm{p}<.001$ for $67 \% \mathrm{Ss}$ ) better than chance and that $0 \%$ Ss performed significantly $(\mathrm{CR}=$

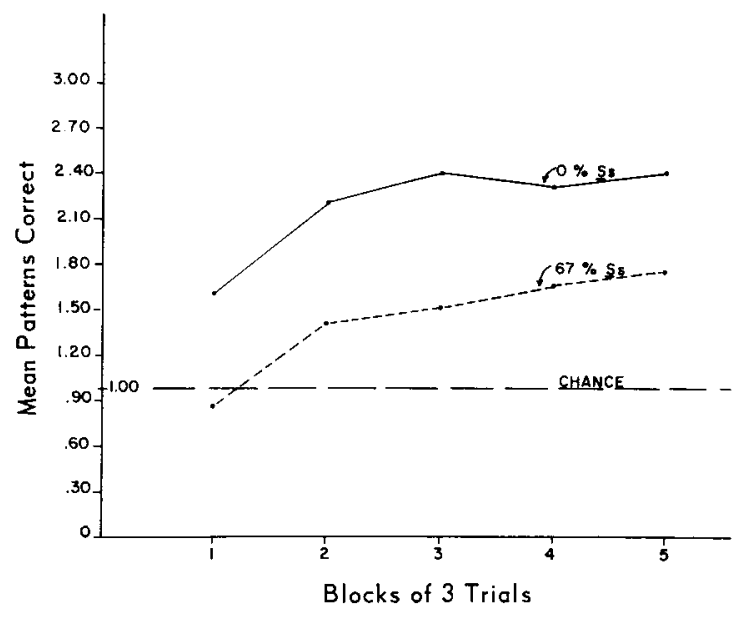

Fig. 1. Block means for $0 \% \mathrm{Ss}$ and $67 \%$ Ss. 
$3.10, \mathrm{p}<.01$ ) more accurately than did $67 \%$ Ss. The $0 \%$ Ss were also superior ( $C R=4.05, p<.001)$ to $67 \%$ Ss in trials 1-9, and to a greater extent than in the last three trials. The $0 \%$ Ss also performed significantly above chance on the first three trials.

Figure 1 indicates that the overall improvement of both experimental groups was approximately the same even though $0 \%$ Ss rapidly reached and maintained a high level of performance while the performance of $67 \%$ Ss improved more slowly. These findings appear to be primarily attributable to the differential requirements imposed by the two experimental tasks.

The $0 \%$ Ss had only to detect the presence of some schema in two of the patterns in order to distinguish them from the random patterns. Indeed, the performance of the $0 \%$ group on trials 1-3 suggests that, even though the instructions did not specify the dimensions along which differences were to be sought, the differences between presence and absence of a schema was sufficiently striking to induce Ss to use that dimension immediately.

The slower improvement of the $67 \%$ group probably resulted from the necessity to learn the specific characteristics of the schema associated with PS1 in order to distinguish the two PS1 patterns from the patterns having a different schema. At the end of 15 trials, however, the $67 \%$ group was still improving, while the $0 \%$ group appears to have leveled off. Thus, a substantially greater number of trials might have allowed the $67 \%$ group to catch up to the $0 \%$ group.

The results indicate that with knowledge of results, Ss can readily learn to distinguish between the presence and the absence of a schema and to distinguish between different schemata. The results also suggest that Ss may spontaneously distinguish between the presence and the absence of a schema even without knowledge-ofresults training.

A previous study (Evans, 1964) with a different task indicated that $\mathrm{Ss}$ could also spontaneously distinguish between two different schemata without knowledge of results, and another study (Edmonds \& Evans, 1966) indicated that in a memory task Ss learned a single schema without knowledge-of-results. Thus, as sug- gested by Attneave (1957), schema learning may play a role in a theory of perceptual learning. The Gibsons (Gibson \& Gibson, 1955; Gibson, 1959) have included in their theory a process of differentiation of higher order variables without knowledge-of-results. If a schema may be regarded as a kind of higher order variable, this theory appears to receive support from these studies of schema learning.

In another frame of reference, a schema may be regarded as a statistical concept (i.e., a concept which is not defined in terms of a few infallible attributes, but in terms of a larger number of fallible attributes which are statistically associated and sufficiently numerous to allow a highly reliable assignment to category). The present results may be regarded as demonstrating that humans readily learn statistical concepts. Quite possibly, many familiar concepts are statistical in the above sense. The recognition of hand printed letters, for example, appears to be based on statistical concepts; a number of computer programs for this purpose make use of statistical decision processes (see Uhr, 1962). The present results suggest that statistical concept formation deserves more attention than it has so far received.

\section{References}

Attneave, F. Transfer of experience with a class-schema to identification-learning of patterns and shapes. J. exp. Psychol., 1957, $54,81-88$.

Edmonds, E. M., \& Evans, S. H. Schema learning without a prototype. Psychon. Sci., 1966, 5, 247-248.

Evans, S. H. A model for perceptual category formation. Unpublished doctoral dissertation, Texas Christian University, 1964.

Evans, S. H., Edmonds, E. M., \& Mueller, M. R. Computer patterns for perception studies: The VARGUS series. Paper presented at Southwestern Psychological Association, April, 1966.

Gibson, J. J. Perception as a function of stimulation. In S. Koch (Ed.), Psychology: a study of a science. Vol. 1. New York: McGraw-Hill, 1959.

Gibson, J. J., \& Gibson, E. J. Perceptual learning: differentiation or enrichment? Psychol. Re'r., 1955a, 62, 32-41.

oldfield, R. C. Memory mechanisms and the theory of schemata. Brit. J. Psychol., 1954, 45, 14-23.

Uhr, L. Pattern recognition computers as models for form perception. Psychol. Bull., 1963, 60, 40-73.

Woodworth, R. S. Experimental psychology. New York: Holt, 1938 